\title{
Design of exercise test, with special reference to heart patients
}

\author{
Hans Åström and Bengt Jonsson \\ From the Departments of Clinical Physiology and Cardiology, Thoracic clinics, Karolinska sjukhuset, Stockholm, \\ Sweden
}

Estimation of exercise tolerance in patients with heart disease and significant symptoms can usually not be expressed in terms of maximal oxygen uptake or power at a fixed heart rate calculated from a 'steady state' exercise test.

The exercise tolerance can be expressed only by the maximal power developed, when limited by symptoms. For this purpose the level of tolerance is better assessed by a test with small increments and short duration of each work load.

In clinical practice this type of almost continuous increase in load was found to be particularly useful in patients with ischaemic heart disease.

Comparative studies showed that the work per heart beat at equal loads is significantly higher in the test with continuous increase in load than in the test with steps of 6 minutes duration, both in normal subjects and in heart patients. The difference, however, is small (7\%) and for practical purposes these tests have equal validity as a measure of the circulatory capacity.

Evaluation of the exercise tolerance from the history alone is often unreliable in patients with heart disease. Some patients with low capacity neglect symptoms. This is often observed in patients who are disabled since childhood and therefore have no experience of a normal circulatory function. Other patients exaggerate their symptoms or suffer from symptoms not associated with a low circulatory capacity. We therefore need methods for the objective determination of physical capacity. Exercise testing is also of great value as a tool to measure the effect of medical and surgical treatment. A simple procedure is to observe the patient climbing stairs. This is, however, not satisfactory as the work load is not well defined and objective recordings of circulatory variables cannot easily be obtained. Standardized exercise tests of different types have been used. The work must involve large muscle groups in order to give information of the oxygen transport capacity. Both treadmill and bicycle ergometer work fulfil this requirement. We prefer work on a bicycle ergometer as this can be accurately calibrated and thus the work intensity can easily be measured without determination of the oxygen consumption, and

Received 29 May 1975. most patients, at least in Sweden, are familiar with pedalling a bicycle.

In healthy subjects the maximal aerobic power is determined by measuring the maximal oxygen uptake or the power developed when the maximal heart rate is obtained. Any further increase in work load will not result in a higher oxygen uptake or heart rate. Patients with severe heart disease are unable to perform such a maximal exercise test and, therefore, various types of submaximal exercise test have been devised. The report of a WHO meeting (World Health Organization, 1968) concerning exercise tests refers to four designs: type 1) single level load, type 2) discontinuous series of increasing loads with intermittent rest periods, type 3) nearly continuous increase in load, type 4) continuous series of increasing loads with an almost steady state at each level, each lasting at least 4 minutes (Fig. 1).

A patient with heart disease should not be suddenly exposed to a load close to his maximal tolerance and type 1, therefore, is not appropriate. Type 2 is tedious and at the highest level of work the state is suddenly changed from rest to a heavy load. Those most suitable for heart patients are types 3 and 4. To determine the aerobic power it is important to use a test that allows the patient to 


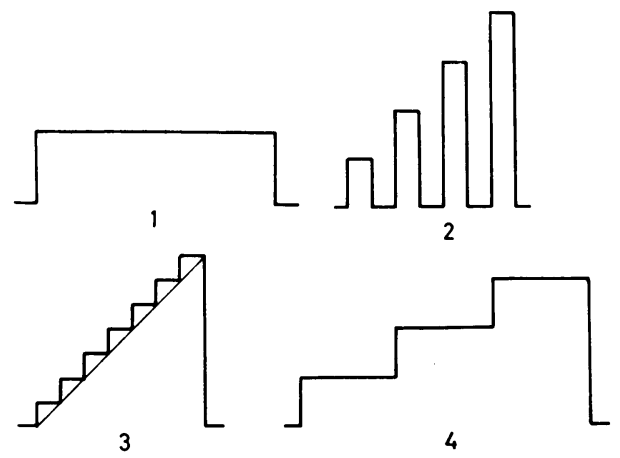

FIG. 1 Schematic drawing of different designs of exercise tests. 1) Single level load. 2) Discontinuous series of increasing loads with intermittent rest periods. 3) Continuous or nearly continuous increase in load. 4) Continuous series of increasing loads with an almost steady state at each level.

reach a steady state. This has been supposed to occur in the type 4. In Sweden this type of test has been widely used in clinical practice for nearly 30 years (Sjöstrand, 1947; Wahlund, 1948). The work lasts for 6 minutes at each load, and with 3 or 4 equal increments the subject should reach a heart rate of about 170 beats per minute. Using the linear relation between load and heart rate, the work at heart rate $170 / \mathrm{min}$ is calculated and the exercise performance is expressed as the power (in Watts or $\mathrm{kpm} / \mathrm{min}$ ) at rate 170 . In older patients the maximal heart rate is lower than this and a reference rate of 130 or 150 has been used (Strandell, 1964). Sometimes the work is stopped before completion of the 6-minute exercise period. The exercise tolerance then is not indicated by the highest power only but also by the working time. A patient who is able to work for 6 minutes at a certain load plainly has a better tolerance than the one who stops the same work after 2 minutes, though the same power output is achieved. In this case Strandell predicted the 'maximal' power by adding a figure proportional to the completed part of the period at the next higher load (Strandell, 1964).

For some years the type 3 work test, with small increments and each step lasting one minute, has also been used in our department. The purpose of this report is to compare tests of type 3 and type 4 , in order to define the relative merits of each.

\section{Subjects and methods}

The usefulness of the exercise test with increasing loads, each step lasting for 6 minutes (type 4), was studied in heart patients referred to the laboratory for preoperative evaluation. From the records a consecutive series of 100 patients was selected. These patients with valvular lesions, congenital heart defects, or coronary heart disease had a wide range of exercise capacities.

A comparison between exercise tests of type 3 and type 4 was made in two groups of healthy subjects. The first group included 7 men and 7 women from the hospital staff, aged between 25 and 57 years, The second group consisted of 24 men, aged 18 to 20 years, entering military service. This comparison was also made in 28 patients with ischaemic heart disease and 23 patients with various acquired and congenital heart diseases. All these patients were referred to the department for preoperative evaluation and were suffering from significant symptoms.

The effect of total duration of work using the type 3 test was also studied in the 14 healthy members of the hospital staff, by performing two tests, one with increments of load twice the other, resulting in half the working time.

All tests were performed in the sitting position on an electrically braked bicycle ergometer (Elema). A 12-lead electrocardiogram was recorded before and after the test, and during the work period 6 chest leads were recorded continuously. Heart rate was counted from the electrocardiogram at the end of each period. In the healthy subjects the work load was increased to exhaustion, but it was not determined whether levelling off of oxygen uptake or heart rate had occurred. In the patients with heart disease the work load was increased until significant symptoms occurred. Most patients stopped the work when they were unable to continue, but in ischaemic heart disease the patients were asked to stop when the chest pain was of the degree they were accustomed to tolerate during their daily activity. In patients with aortic stenosis the test was ended at the appearance of symptoms and not continued to maximal tolerance if the patient suffered from angina, dyspnoea, or dizziness.

When the two types of test were compared in the same patient, exercise was stopped at about the same severity of symptoms. A doctor with wide experience of exercise tests was present during the procedure and evaluated the symptoms and the signs of strain. In all studies where each individual performed two tests, these were done on two consecutive days at the same time of the day and on the same ergometer. The order of the tests was ramdomizad.

'Maximal power' (Strandell, 1964) was calculated by adding to the maximum work load at which the subject completed 6 minutes of exercise a proportion of the next increment of load corresponding to the proportion of the 6-minute exercise period completed. ${ }^{1}$

Student's paired t-test was used to test the significance of differences between mean values.

\footnotetext{
${ }^{1}$ For example, if a patient completes a 6-minute exercise period at 60 Watts and then stops after 3 minutes at 90 Watts, the 'maximal power' is $60+\left(\frac{3}{6} \times 30\right)=75$ Watts.
} 


\section{Results}

Continuous exercise with increasing loads and steps of 6 minutes duration (type 4)

The original design of the test required that a steady state should be obtained, and at the highest load the heart rate should be close to 170 per minute. These two criteria were tested in 100 heart patients. From Fig. 2 it is evident that very few patients reached a steady state of the heart rate.

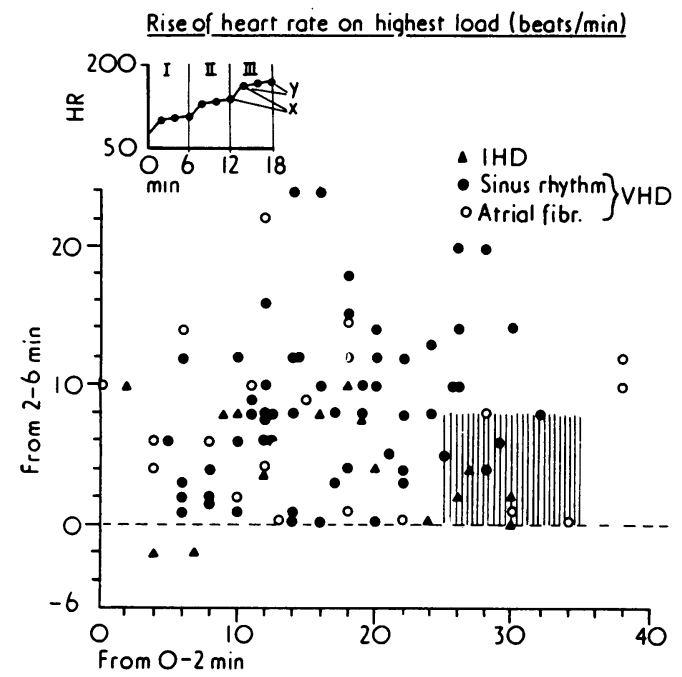

FIG. 2 Exercise test with steps of 6 minutes duration in 100 patients with heart disease. Increase in heart rate from 2 to 6 minutes of work on the highest load (y-axis) in relation to the increase of heart rate during the first 2 minutes of work on the highest load $(x$-axis). The schematic diagram in the upper left part of the figure represents an ideal heart rate response, with a 'steady state' at each work level, and heart rate reaching 170/min after three increments of work load. Patients with this type of response will be located within the shaded area of the main diagram. Very few patients are in a 'steady state'.

An ideal test should follow a pattern indicated in the upper right part of the diagram. Assessment of a steady state has been defined as an increase in heart rate of less than 10 beats a minute between the second and sixth minutes (Sjöstrand, 1967). Using these criteria the symbols in the diagram should be located within the shaded area: very few are. From Fig. 3 it is also evident that many patients do not reach a heart rate close to 170 per minute. The patients stopped exercising at lower heart rates because of symptoms, such as respiratory distress, chest pain, general fatigue, or tiredness in the legs. Often the work was stopped before the end of a 6-minute period. An evaluation

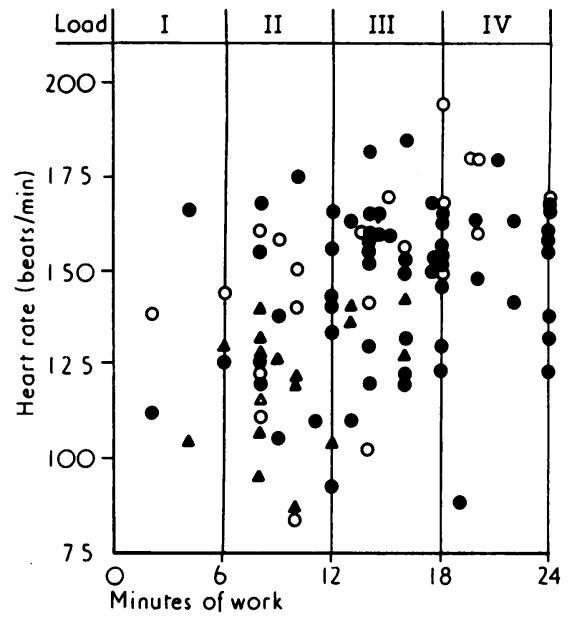

FIG. 3 Exercise test with steps of 6 minutes duration in 100 patients with heart disease. Duration of work ( $x$-axis) in relation to heart rate ( $y$-axis) at the end of work. Some patients stopped exercising on load I and a few could continue to load IV. Many patients ended the work at a heart rate below 150/min. $\Delta$ Ischaemic heart disease.

- Sinus rhythm, $\bigcirc$ atrial fibrillation, in patients with other forms of heart disease.

of the exercise tolerance according to the original design of the test was, therefore, possible only in a few patients.

Comparison between tests with steps of 6 minutes duration (type 4) and 1 minute duration (type 3 ) in healthy subjects

Performance in the two tests was expressed as the work-pulse (i.e. work per heart beat) and calculated as:

$$
\frac{\text { Watts } \times 60}{\text { beats/minute }} \text { (Joules/beat) }
$$

This was calculated from the highest load that was completed in both tests to make comparison between the two tests possible, since many subjects were able to work on a higher load in a type 3 test. In the healthy subjects from the hospital staff there was on average a 7 per cent higher work-pulse in the type 3 than in the type 4 test (Fig. 4). The difference was highly significant $(P<0.001)$. The subjects represented a wide range of physical fitness, with a variation of work-pulse from $36 \cdot 0$ to $102 \cdot 1 \mathrm{~J} /$ beat. The mean duration of work at type 4 test was 22 minutes and at type 3 test 14 minutes. The work-pulse in the young men on military service varied between 46.2 and 81.6 Joules (Fig. 5). The average work-pulse for the type 3 test was 64.8 and for the type 4 test 


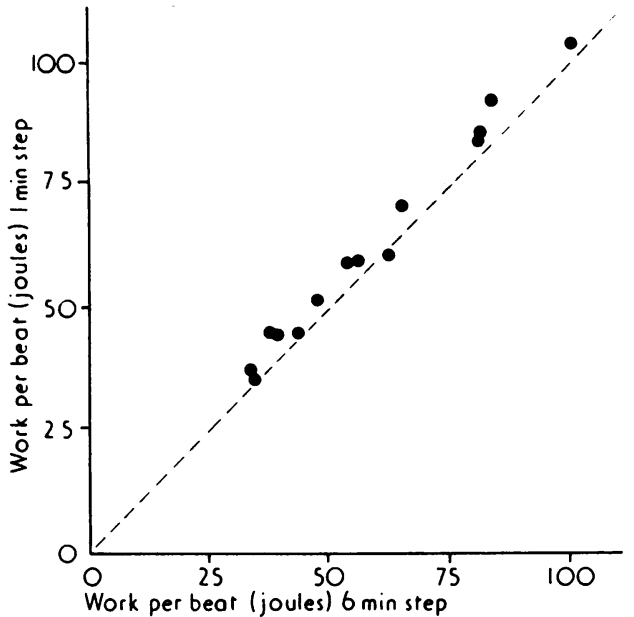

FIG. 4 Relation between tests with steps of 6 minutes duration ( $x$-axis) and 1 minute duration (y-axis) in 14 healthy subjects aged 25 to 57 years. The exercise performance is expressed in work per heart beat.

$60.0 \mathrm{~J} /$ beat. This difference of 8 per cent was highly significant $(P<0.001)$. The mean duration of the two tests was 15 and 16 minutes, respectively; this difference is not significant.

\section{Effect of duration of work on heart rate}

The increase of the load for each step must vary according to the subject's work capacity. If equal steps were used for all subjects the total working time

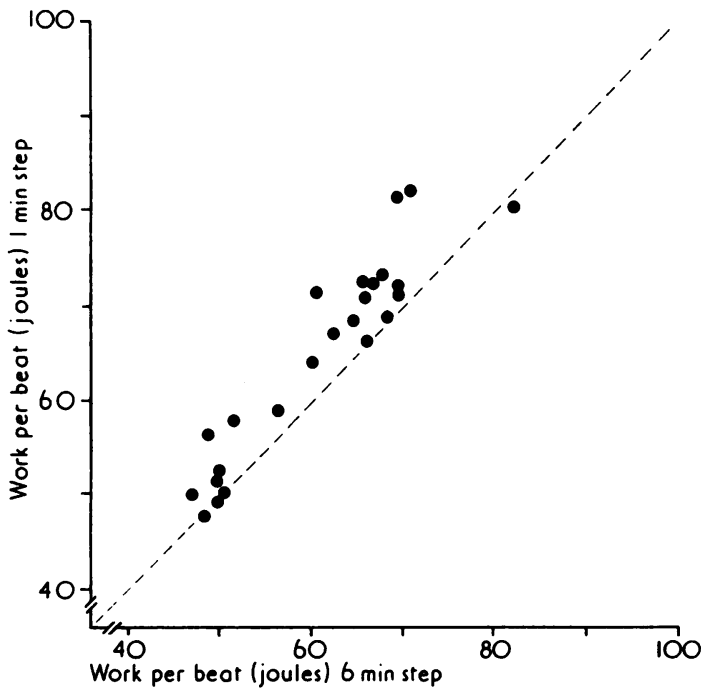

F IG. 5 The same relation as in Fig. 4 in 24 healthy men aged 18 to 20 years.

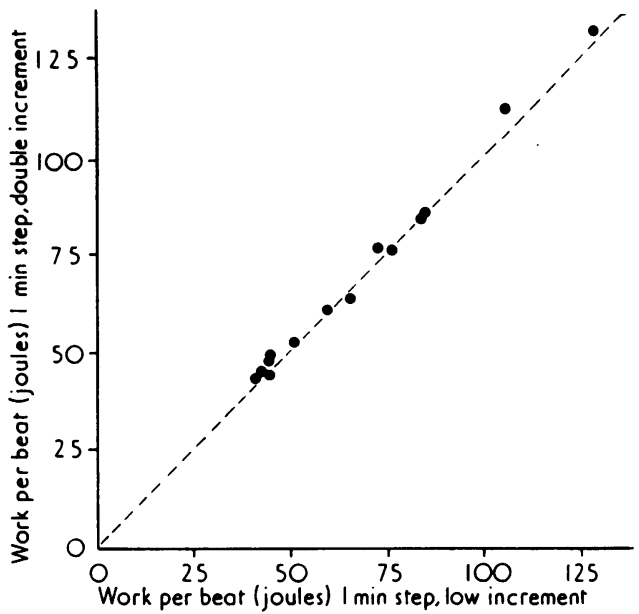

FIG. 6 Relation between two work tests with steps of 1 minute duration in 14 healthy subjects. The $y$-axis represents tests with increments of each step twice that of the $x$-axis. The total duration of work during tests on the $y$-axis are half of the tests on the $x$-axis.

would be very short for some and in others very long. It is, however, complicated to standardize a test where all individuals will reach the highest load within a predicted time (Arstila, 1972). The effect of variation in total working time by difference in increments of load is shown in Fig. 6. No difference of work-pulse was observed. The total work performed at low increments was $88.0 \mathrm{~kJ}$ and at high increments $51.2 \mathrm{~kJ}$. Within this variation of working time, 14 and 7 minutes, respectively, the heart rate is dependent only on the power developed and not on working time or total work performed.

Comparison between tests with steps of 6 minutes duration (type 4) and 1 minute duration (type 3 ) in patients with heart disease

A. Ischaemic heart disease Chest pain was the limiting symptom for exercise performance in all patients. When comparing the work-pulse, the highest load that was completed at both tests was used for the calculation; no difference was found (Fig. 7). The maximal heart rate obtained when the test was ended because of pain was on average 5 per cent higher in type 3 tests than in type $4(P<0.05)$ (Fig. 8). The highest load the patient could achieve was 19 per cent higher with the type 3 test than with type $4(P<0.001)$ (Fig. 9). In type 4 tests some patients had to discontinue exercise before completion of a 6-minute period, in some cases after only 1 minute. In type 3 tests the 1 -minute period was always completed.

If a correction is made for incomplete exercise 


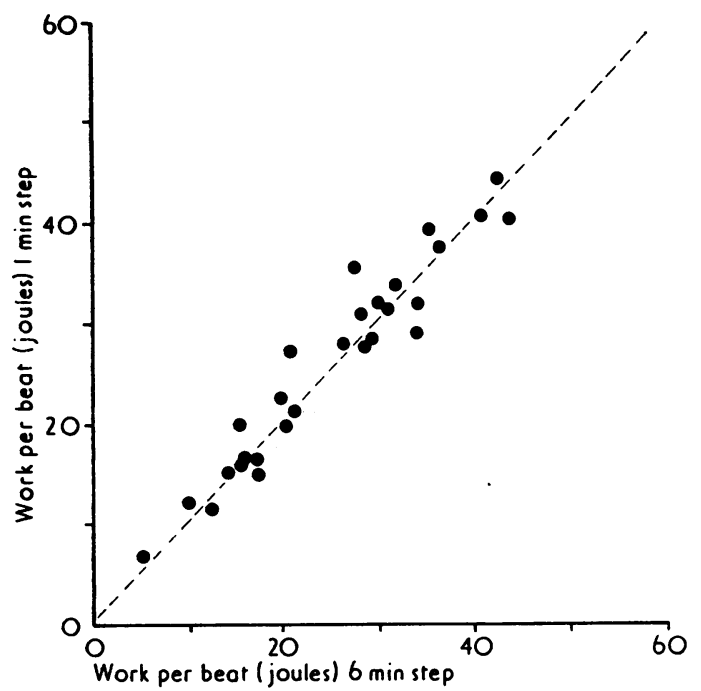

FIG. 7 The same relation as in Fig. 4 in 28 patients with ischaemic heart disease.

periods as suggested by Strandell (1964) in the type 4 tests, the difference between the two tests was greater, type 3 then being 41 per cent higher than type $4(P<0.001)$. The average duration of work was 10.0 minutes for the type 3 tests and 12.6 minutes for type 4 . The average total work performed was $32.2 \mathrm{~kJ}$ for the type 3 tests and $38.4 \mathrm{~kJ}$ for type 4 .

B. Valvular and congenital heart disease The work-pulse, calculated as for patients with ischaemic

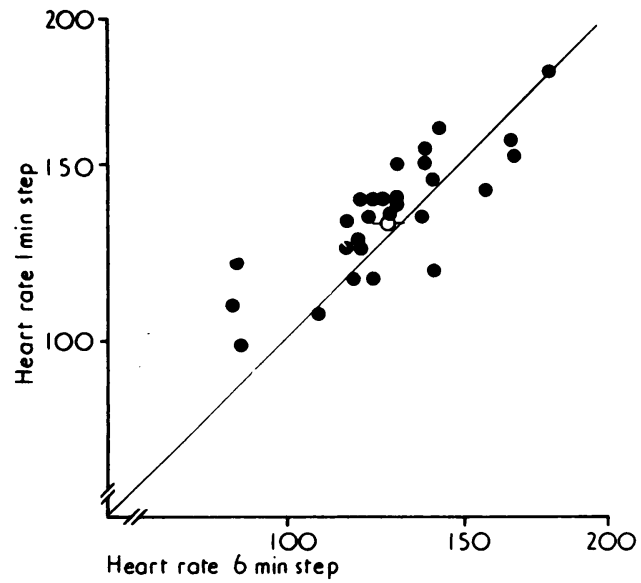

FIG. 8 Relation between highest heart rate obtained during tests with steps of 6 minutes duration ( $x$-axis) and 1 minute duration (y-axis) in 28 patients with ischaemic heart disease. The open circle represents the mean of the group.

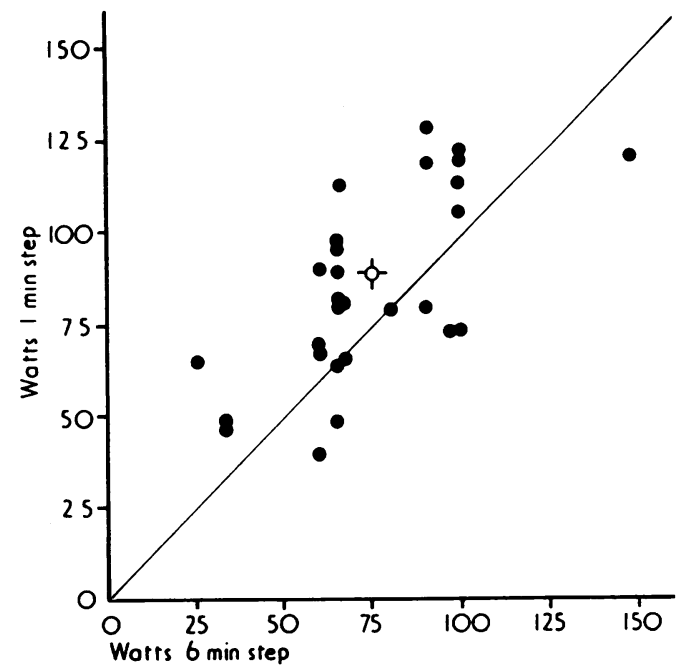

FIG. 9 Relation between highest load achieved during tests with steps of 6 minutes duration ( $x$-axis) and 1 minute duration (y-axis) in 28 patients with ischaemic heart disease. The open circle represents the mean of the group.

heart disease, was on average 7 per cent higher for type 3 tests. This difference was significant ( $P$ $<0.01)$ and more obvious for patients with a work pulse over 60 Joules than for patients with lower values (Fig. 10). The maximal heart rate obtained was on an average 2 per cent higher in type 3 tests than in type 4 tests $(P>0.3)$ (Fig. 11). The highest load achieved was 14 per cent higher during the type 3 test $(P<0.01)$ (Fig. 12). When Strandell's correction was made, the difference was 29 per cent $(P<0.001)$. The average duration of the work was

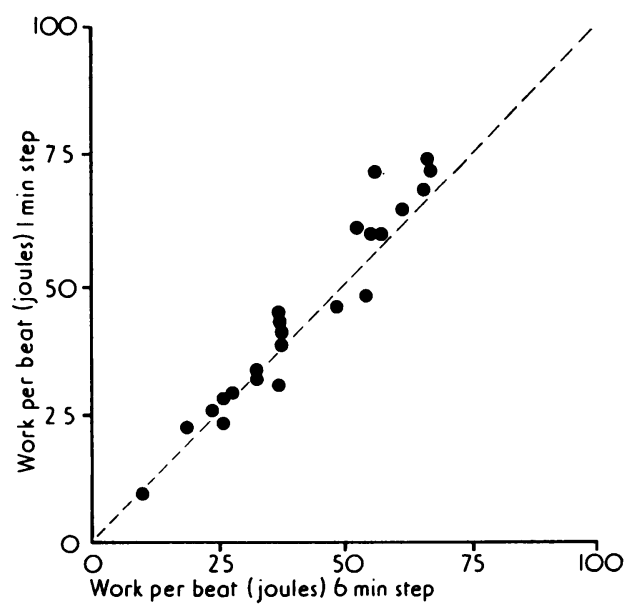

FIG. 10 The same relation as in Fig. 4 in 23 patients with valvular or congenital heart disease. 


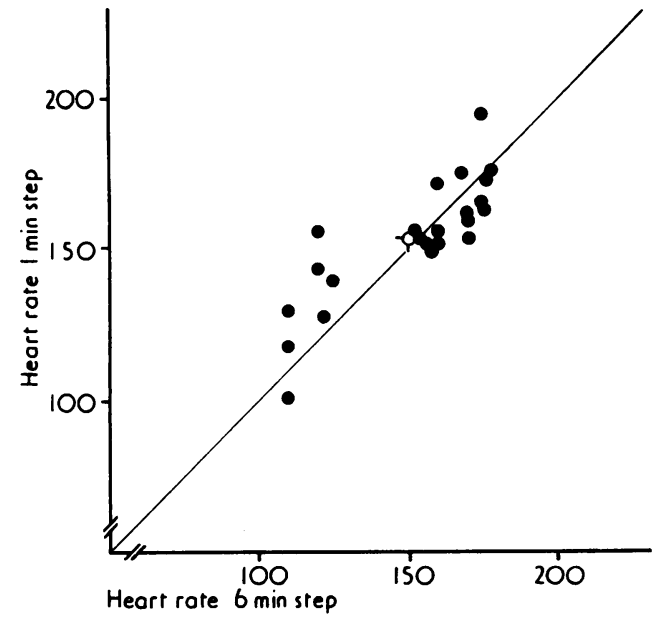

FIG. 11 Relation between highest heart rate obtained during tests with steps of 6 minutes duration $(x$-axis) and 1 minute duration (y-axis) in 23 patients with valvular or congenital heart disease. The open circle represents the mean of the group.

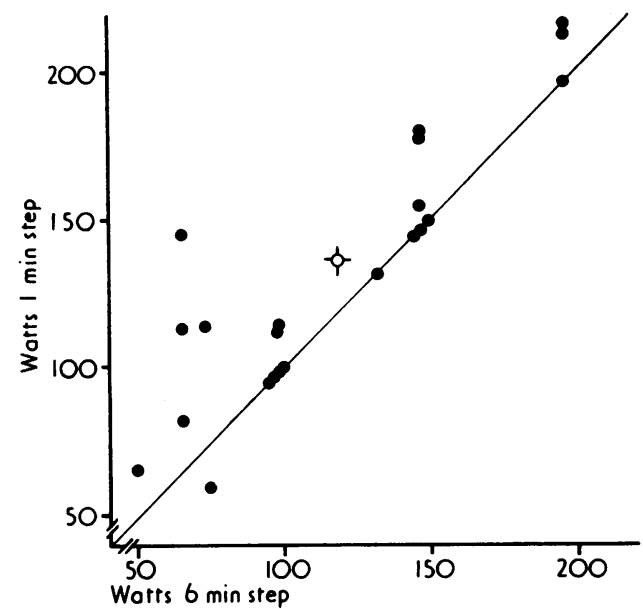

FIG. 12 Relation between highest load achieved during tests with steps of 6 minutes duration ( $x$-axis) and 1 minute duration (y-axis) in 23 patients with valvular or congenital heart disease. The open circle represents the mean of the group.

7.8 minutes for the type 3 test and 14.3 minutes for the type 4 test.

\section{Discussion}

An exercise test must be adapted to the subjects who are examined and to the purpose of the test. For estimation of the maximal aerobic power the maximal oxygen uptake or the power developed (steady state) at the maximal heart rate must be measured. This test cannot be used in patients with severe heart disease. The term 'maximal working capacity' is, however, sometimes used also in clinical tests without any objective definition. Often it is used for the work load before the patient has to discontinue exercise on account of various symptoms, even when this occurs at rather low heart rates. This does not indicate the maximal circulatory capacity and the term 'maximal working capacity' should not be used.

The power at a given submaximal heart rate, e.g. 170 beats per minute, is a measure of the circulatory capacity (Sjöstrand, 1967). During work the oxygen uptake increases in a linear relation to the increase of work load and (at high heart rates) the mechanical efficiency is almost constant in different subjects (Asstrand, 1952). The oxygen uptake at heart rate (HR) 170 is a function of the stroke volume (SV) and the arteriovenous oxygen difference (AVD) according to Fick's equation $\left(\mathrm{V}_{2}=\mathrm{HR} \times \mathrm{SV} \times\right.$ AVD). Consequently the power or work-pulse at a heart rate of 170 is also a function of stroke volume and arteriovenous oxygen difference. In healthy individuals a linear relation exists between the power at a heart rate of 170 and such circulatory measurements as heart volume, blood volume (Kjellberg, Rudhe, and Sjöstrand, 1949), stroke volume (Bevegård, Holmgren, and Jonsson, 1963), and maximal oxygen uptake (Åstrand, 1952).

This type of test, however, cannot be used in patients with heart disease and severe symptoms. Because of lack of a steady state and a low heart rate on the highest load, an extrapolation to heart rate 170 has no validity (see Fig. 2). The exercise tolerance can only be expressed by the maximal power obtained when work is ended because of symptoms. The type and degree of limiting symptoms should be described, and the highest heart rate obtained should be indicated. The level of tolerance is best defined by a test with small increments and short duration of each step, to avoid a very long duration of the test. A design with steps lasting 1 minute is appropriate. In patients with low tolerance the total working time then will be 5 to 10 minutes with increments of 10 Watts. If this type of test is compared with the so-called steady state test with 6-minute steps, a very small difference is found in work-pulse both in healthy subjects and a group of patients with heart disease with a wide range of exercise tolerance. Therefore, both tests are equally valid as a measure of the circulatory function expressed as the power at heart rate 170 .

Most patients with ischaemic heart disease in this study had a low exercise tolerance. The difference in duration of work in the two types of test was small. The patients continued the work to a slightly 
higher heart rate $(5 \%)$ and a higher load $(19 \%)$ during the test with steps of 1 minute than during the test with steps of 6 minutes duration. This greater difference for maximal load than for maximal heart rate in spite of equal work-pulse seems contradictory. The work-pulse, however, was calculated from equal loads completed for 6 minutes and 1 minute, respectively, during the two tests. When an additional load is performed during the test with 1-minute steps the heart rate does not rise proportionally to the increase in work-pulse, with increasing load.

Although the maximal power for the test with 6-minute steps was smaller than for 1-minute steps, the total work performed was significantly greater. This may explain the lower maximal load obtained. Measuring total work as an index of exercise tolerance of rather short duration is, however, useless both in normal subjects and, even more, in patients with heart disease. Measurements of power or work per heart beat give a better indication of myocardial stress than working time per se. Thus, a wide range of total work can be achieved in the same patient, depending on the magnitude of load and the duration of the test.

In patients with angina pectoris, the exercise tolerance is defined as the power developed when chest pain occurs, which is equal in character and severity to what the patient is used to in his daily life. This level is best measured by a test with a continuous or almost continuous increase of the load. Then the tolerance can be expressed as power developed without correction for working time. The duration of the test should be more than 3 minutes (Redwood et al., 1971) and should not exceed 15 to 20 minutes. A continuous increment of 10 Watts every minute will satisfy this in most patients, as very few will perform more than 150 to 200 Watts. When the effect of treatment is studied the same design should be used for the test before and after treatment of each patient. The reproducibility of a single exercise test with 1 -minute steps, calculated from duplicate determinations performed within 4 days in a group of patients with similar severity of coronary artery disease was found to be 6.6 per cent ( $\AA$ ström and Vallin, 1974).

A further advantage of the continuous type of test is the low starting load where the first loads can be looked upon as a warming-up period. The critical choice of the correct increments of load is also avoided, when too large increments might be dangerous for the patient.

Though both types of test seem to give about the same information, the type 3 test with 1-minute steps is more suitable for patients with low physical capacity, and furthermore is less time consuming. It can be completed in less than half the time required for the test with 6-minute steps.

\section{Conclusions}

In patients with heart disease and significant symptoms an estimation of exercise tolerance can usually not be expressed in terms of power at heart rate 170 beats per minute using a test with increasing loads each lasting for 6 minutes. Most patients stop working at much lower heart rates because of various symptoms, and a steady state is not obtained. The exercise tolerance is better defined by a test with continuous or nearly continuous increase in load.

In normal subjects the work performed per heart beat (work-pulse) at equal loads is significantly higher in the test with nearly continuous increase in load than in the test with steps of 6 minutes duration. The difference, however, is small and for practical purposes both tests have equal validity, even as a measure of the circulatory capacity expressed as the power at heart rate 170 , both in healthy subjects and patients with heart disease.

If the working time is less than 15 minutes, a variation of the duration to half that time does not influence the work-pulse. Furthermore, the heart rate is determined by power and not by total work or duration of the test.

\section{References}

Arstila, M. (1972). Pulse-conducted triangular exercise-ECG test. Acta Medica Scandinavica, Suppl. 529.

Åstrand, P.-O. (1952). Experimental Studies of Physical Working Capacity in Relation to Sex and Age. Munksgaard, Copenhagen.

Åström, H., and Vallin, H. (1974). Effect of a new betaadrenergic blocking agent, ICI 66082, on exercise haemodynamics and airway resistance in angina pectoris. British Heart fournal, 36, 1194.

Bevegård, S., Holmgren, A., and Jonsson, B. (1963). Circulatory studies in well trained athletes at rest and during heavy exercise, with special reference to stroke volume and the influence of body position. Acta Physiologica Scandinavica, $\mathbf{5 7}, 26$.

Kjellberg, S. R., Rudhe, U., and Sjöstrand, T. (1949). The relation of the cardiac volume to the weight and surface area of the body, the blood volume and the physical capacity for work. Acta Radiologica, 31, 113.

Redwood, D. R., Rosing, D. R., Goldstein, R. E., Beiser, G. D., and Epstein, S. E. (1971). Importance of the design of an exercise protocol in the evaluation of patients with angina pectoris. Circulation, 43, 618.

Sjöstrand, T. (1947). Changes in the respiratory organs of workmen at an ore melting works. Acta Medica Scandinavica, Suppl. 196, 687. 
Sjöstrand, T. (1967). Clinical Physiology, p. 523. Lippincott, Phildelphia.

Strandell, T. (1964). Circulatory studies on healthy old men. Acta Medica Scandinavica, Suppl. 414.

Wahlund, H. (1948). Determination of the physical working capacity. Acta Medica Scandinavica, Suppl. 215.
World Health Organization (1968). Exercise tests in relation to cardiovascular function. WHO Technical Report Series, No. 388, Geneva.

Requests for reprints to Dr. H. Aström, Klin Fys Lab, Huddinge Sjukhus, S-141 86 Huddinge, Sweden. 